\title{
PERCEPÇÕES DE MULHERES SOBRE A ASSISTÊNCIA DE ENFERMAGEM DURANTE O PARTO NORMAL
}

\author{
WOMEN'S PERCEPTIONS ABOUT NURSING \\ CARE DURING NORMAL DELIVERY
}

\section{PERCEPCIONES DE LAS MUJERES SOBRE LA ATENCIÓN DE ENFERMERÍA DURANTE EL PARTO NORMAL}

\author{
Aiara Nascimento Amaral Bomfim ${ }^{1}$ \\ Telmara Menezes Couto ${ }^{2}$ \\ Keury Thaisana Rodrigues dos Santos Lima ${ }^{3}$ \\ Lais Teixeira da Silva Almeida ${ }^{4}$ \\ Gleice de Oliveira Santos ${ }^{5}$ \\ Ariane Teixeira de Santana ${ }^{6}$
}

Como citar este artigo: Bomfim ANA, Couto TM, Lima KTRS, Almeida LTS, Santo GO, Santana AT. Percepções de mulheres sobre a assistência de Enfermagem durante o parto normal. Rev baiana enferm. 2021;35:e39087.

Objetivo: conhecer a percepção de mulheres sobre a assistência de Enfermagem recebida durante o processo de parto normal. Método: pesquisa descritiva com abordagem qualitativa, efetuada em duas maternidades públicas de Salvador, Bahia, Brasil. A coleta de dados foi efetuada por entrevista, entre os meses de novembro de 2017 e abril de 2018. Participaram da pesquisa 13 mulheres. A sistematização dos dados foi realizada pelo referencial teórico de Bardin. Resultados: emergiram duas categorias científicas: assistência de Enfermagem permeada por satisfação; e assistência permeada por relações verticais e sentimentos de abandono. As mulheres verbalizaram satisfação com a assistência de Enfermagem relacionadas à aplicação dos métodos não farmacológicos para alívio da dor, apoio e promoção do bem-estar, embora também se fez presente a verticalização das relações e a ausência de acompanhamento profissional. Conclusão: a percepção das mulheres sobre a assistência de Enfermagem recebida durante o processo de parto normal foi dicotômica.

Descritores: Assistência de Enfermagem. Assistência ao Parto. Enfermagem Obstétrica. Parto Normal. Parto Humanizado.

Objective: to know women's perception about the nursing care received during the normal delivery process. Method: descriptive research with qualitative approach, carried out in two public maternity hospitals in Salvador, Bahia, Brazil. Data were collected by interview between November 2017 and April 2018. The participants were 13 women. Data systematization was performed by Bardin's theoretical framework. Results: two scientific categories emerged: Nursing care permeated by satisfaction; and care permeated by vertical relationships and feelings of abandonment. The women verbalized satisfaction with Nursing care, related to the application of non-pharmacological methods for pain relief, support and promotion of well-being, despite the vertical-oriented process of relationships and the absence

\footnotetext{
Enfermeira. Mestre em Enfermagem. Professora do Centro Universitário Jorge Amado. Salvador, Bahia, Brasil. aiaraamaral@hotmail.com. http://orcid.org/0000$0001-9262-0984$.

Enfermeira. Doutora em Enfermagem. Professora da Universidade Federal da Bahia. Salvador, Bahia, Brasil. http://orcid.org/0000-000 I-6836-8563.

Enfermeira. Mestre em Enfermagem e Saúde. Universidade Federal da Bahia. Salvador, Bahia, Brasil. http://orcid.org/0000-0003-0768-I I04

Enfermeira. Mestre em Enfermagem e Saúde. Enfermeira Obstetra da Maternidade João Batista Caribé. Salvador, Bahia, Brasil. http://orcid.org/0000-0002$7582-8465$.

Enfermeira. Universidade Federal da Bahia. Salvador, Bahia, Brasil. http://orcid.org/0000-0003-3358-6004.

6 Enfermeira. Especialista em Obstetrícia. Universidade Federal da Bahia. Salvador, Bahia, Brasil. http://orcid.org/0000-000 I-6264-7| I5.
} 
of professional follow-up. Conclusion: women's perception about Nursing care received during the normal delivery process was dichotomous.

Descriptors: Nursing Care. Childbirth Assistance. Obstetric Nursing. Normal Childbirth. Humanized Childbirth.

Objetivo: conocer la percepción de las mujeres sobre la atención de enfermería recibida durante el proceso normal de parto. Método: investigación descriptiva con enfoque cualitativo, realizada en dos hospitales públicos de maternidad en Salvador, Bahia, Brasil. Los datos fueron recogidos por entrevista entre noviembre de 2017 y abril de 2018. Participaron en el estudio 13 mujeres. La sistematización de datos fue realizada por el marco teórico de Bardin. Resultados: surgieron dos categorías científicas: cuidados de enfermería impregnados de satisfacción; y la asistencia impregnada de relaciones verticales y sentimientos de abandono. Las mujeres verbalizaron la satisfacción con la atención de enfermería, relacionada con la aplicación de métodos no farmacológicos para el alivio del dolor, el apoyo y la promoción del bienestar, aunque también estuvieron presentes la verticalización de las relaciones y la ausencia de seguimiento profesional. Conclusión: la percepción de las mujeres sobre la atención de enfermería recibida durante el proceso normal de parto fue dicotómica.

Descriptores: Cuidado de Enfermería. Asistencia al Parto. Enfermería Obstétrica. Parto Normal. Parto Humanizado.

\section{Introdução}

A assistência às mulheres na obstetrícia vivencia mudanças nos últimos vinte anos, principalmente quando se refere à atenção ao parto e nascimento. Essas mudanças implicaram em redução de 44\% dos óbitos de mulheres em idade fértil no mundo nos anos de 1990 a $2015^{(1)}$. A assistência às mulheres prestada por enfermeiras faz parte desse contexto de avanços, visto que a atuação dessas profissionais, baseada na humanização da assistência ao parto e nascimento, está intimamente relacionada às mudanças nas práticas assistenciais que institucionalizaram o processo de gestar e parir, podendo contribuir sobremaneira para o empoderamento dessas mulheres durante a parturição ${ }^{(2)}$.

A assistência da enfermeira obstetra reforça a importante contribuição dessa profissional no que tange à prática assistencial, em conformidade com o preconizado pela assistência humanizada ao parto e nascimento. Essa assistência mostra-se capaz de reconfigurar o modelo de atenção ao parto, além de contribuir com mudanças mediante uma atuação autônoma, colaborativa, de qualidade, em atendimento às políticas públicas de saúde nacionais e internacionais ${ }^{(3)}$.

Os gestores de saúde devem proporcionar condições para a implementação do modelo de assistência que incluam a enfermeira obstetra e a obstetriz na assistência ao parto de baixo risco, por apresentar vantagens em relação à redução de intervenções e propiciar maior satisfação das mulheres. A transformação do modelo assistencial obstétrico é um desafio atual que requer esforços tanto de gestores quanto dos profissionais de saúde ${ }^{(4)}$.

O motivo de muitas mulheres temerem o parto normal é o grande número de intervenções, muitas vezes desnecessárias, que rodeiam a assistência obstétrica: jejum prolongado, acesso venoso, medicamentos indutores do trabalho de parto, manobra de kristeller, episiotomia dentre outras. Dessa forma, somado ao medo da dor e a afirmativas que questionam a capacidade das mulheres parirem, a cesariana configura-se como um acalento ${ }^{(5)}$. Tal cenário pode ter influenciado no aumento do índice de cesariana nos últimos anos no Brasil.

Desse modo, com o intuito de fortalecer a humanização da assistência ao parto, seja ele por via vaginal ou não, faz-se necessário incluir a mulher como centro do processo de parto, respeitando e incentivando a sua autonomia e liberdade de escolha em momentos decisórios. Portanto, é de extrema relevância reconhecer a atuação da enfermeira nesse cenário, pois essa profissional traz em sua essência uma assistência que transcende o uso de procedimentos técnicos e envolve sensibilidade ${ }^{(6)}$. 
A atuação da Enfermagem obstétrica na melhora do cuidado é demonstrada em diversos estudos e é reconhecida pela Organização Mundial de Saúde (OMS) como possibilidade para a redução de procedimentos desnecessários, bem como redução da morbimortalidade perinatal ${ }^{(1-7)}$. Ademais, algumas estratégias foram lançadas nos últimos anos para disseminar o processo de humanização do parto e nascimento. Dessa forma, surgiu o seguinte questionamento: como as mulheres percebem a assistência dessa categoria profissional? Portanto, esta pesquisa objetiva conhecer a percepção de mulheres sobre a assistência de Enfermagem recebida durante o processo de parto normal.

\section{Método}

Trata-se de pesquisa descritiva, exploratória e de abordagem qualitativa, vinculada ao projeto matriz "Atores sociais e fatores envolvidos no processo de parto", desenvolvida em duas maternidades públicas. Tendo em vista que o primeiro local de estudo entrou em reforma recebendo somente pacientes reguladas clinicamente com alto risco, solicitamos ao Comitê de Ética em Pesquisa (CEP) expansão do local de coleta de dados. Ambas as instituições são acadêmicas assistenciais com perfis similares de usuárias, localizadas na cidade de Salvador, Bahia, Brasil.

Esta pesquisa foi inserida na Plataforma Brasil e avaliada pelo Comitê de Ética em Pesquisa, obtendo Parecer favorável consubstanciado n. 2026663/2017. Os componentes éticos e legais foram respeitados em todas as etapas da pesquisa, em conformidade com a Resolução n. 466/12 do Conselho Nacional de Saúde (CNS). As participantes foram abordadas e convidadas a participarem da pesquisa; em seguida houve a assinatura do Termo de Consentimento Livre e Esclarecido (TCLE), contendo informações sobre as medidas que asseguravam o anonimato e a confidencialidade sobre a origem dos dados obtidos. As participantes foram identificadas com a letra "M" de mulher, seguida de um número cardinal, relacionado à ordem de ocorrência da entrevista: M1, M2 até M13.
As participantes da pesquisa foram escolhidas aleatoriamente, mediante os seguintes critérios de inclusão: puérperas $24 \mathrm{~h}$ pós-parto normal, com parto assistido predominantemente por enfermeira obstetra, confirmado em prontuário, e que receberam assistência durante o trabalho de parto e parto nas maternidades pesquisadas. Foram excluídas as puérperas menores de 18 anos, que tiveram parto durante o percurso à maternidade, ou reguladas de outra instituição. A interrupção da coleta de dados e a definição do tamanho da amostra selecionada ocorreram com a saturação teórica na décima terceira entrevista.

O período de coleta de dados ocorreu entre os meses de novembro de 2017 e abril de 2018. A técnica escolhida para a coleta foi a entrevista, por proporcionar menor distorção da realidade a ser pesquisada, devido à sua amplitude e flexibilidade. O instrumento utilizado foi um formulário semiestruturado. A primeira parte do formulário foi destinada à caracterização sociodemográfica e obstétrica das participantes e a segunda parte foi a própria questão norteadora: como foi a assistência recebida pela Enfermagem durante todo o processo do seu parto?

As entrevistas tiveram duração média de 20 minutos e foram gravadas por meio de gravador eletrônico e posteriormente transcritas na íntegra até não serem identificadas novas percepções que pudessem acrescentar outras inferências nas unidades de registro. Após a organização dos depoimentos, ocorreu o agrupamento de informações da percepção de mulheres sobre a assistência de Enfermagem recebida durante o processo de parto normal.

Os dados foram interpretados com base no referencial teórico Análise de Conteúdo, proposta por Bardin ${ }^{(8)}$. Essa técnica passa por etapas essenciais para a compreensão e melhor exploração do material coletado mediante a leitura dos discursos das entrevistadas e aglomeração em núcleos das ideias centrais da fala de cada uma.

Inicialmente realizou-se uma pré-análise da leitura flutuante do material. Em seguida, foi efetuada leitura minuciosa, a fim de se familiarizar com o conteúdo e favorecer a construção das categorias. Foram realizados recortes em unidades 
de significado, por meio de análise e seleção dos fragmentos das entrevistas que expressavam a percepção de mulheres sobre a assistência de enfermagem recebida durante o processo de parto normal. Posteriormente, seguiu-se para a última etapa, a da inferência, que possibilitou o tratamento e a interpretação dos resultados, respaldadas por autores que discutem a temática.

Emergiram das entrevistas duas categorias científicas, sendo que a primeira exigiu subcategorização para facilitar a apreensão do conteúdo das falas das participantes: Categoria 1 - Assistência de Enfermagem permeada por satisfação, Categoria 2 - Assistência permeada por relações verticais e sentimentos de abandono.

\section{Resultados}

Participaram da pesquisa 13 mulheres, com idade média de 31 anos. Em relação à escolaridade, dez mulheres afirmaram ter cursado ensino médio completo, duas, o ensino superior e apenas uma mulher possuía o ensino fundamental incompleto. No que tange à situação econômica das participantes, sete possuíam renda individual e seis estavam desempregadas. A renda familiar variou de $1 / 5$ a 6 salários mínimos; a cor parda foi predominante. E quanto ao número de gestações, a média foi de três gestações por mulher.

\section{Categoria 1- Assistência de Enfermagem permeada por satisfação}

As participantes do estudo relatam satisfação com a assistência de Enfermagem relacionadas ao atendimento recebido, à promoção do bem-estar que lhes foram proporcionadas e com o desenvolvimento de relação de confiança.

\section{Sub-categoria 1- Atendimento recebido}

As mulheres afirmaram estar satisfeitas com o atendimento de Enfermagem durante o processo de parto. Para tanto, houve relatos de promoção de conforto, tranquilidade e alívio da dor, evidenciados nos depoimentos a seguir:
Eu já fui em outra maternidade, e não fui tão bem atendida como aqui, parece que eu estava em casa. (M1).

A equipe [de enfermagem] me ajudou, me incentivou. o parto daqui foi diferenciado, tipo clínica particular. Eu tive essa impressão, pelo cuidado das pessoas ao meu redor. Fui muito bem atendida. (M2).

Eu achei bom a assistência que recebi. O que eu mais gostei foi que ninguém foi grossa comigo, não fizeram ignorância comigo, falaram com calma. (M10).

[...] quando eu saí da salinha [admissão], eu falei muito do papel dos enfermeiros, que me deram todo esse apoio. Ele foi muito importante para mim. Eu me senti abraçada por ele. Sabe quando você deposita toda a sua confiança naquela pessoa, foi o que aconteceu comigo. (M12).

\section{Sub-categoria 2 - Promoção do bem-estar da mulher}

A assistência de Enfermagem foi frequentemente associada à palavra conforto, denotando uma promoção do bem-estar dessas mulheres:

\begin{abstract}
A enfermeira estava junto a mim, segurando a minha mão, cada dor que eu sentia ela falava pode segurar em mim, pode apertar a minha mão. (M8).

Eu me senti confortável com o tratamento das funcionárias daqui, elas me apoiaram segurando na minha mão, me dando forç. (M11).

A assistência [da enfermagem] foi ótima, porque me atenderam bem, me deram a atenção que eu precisava, auxiliou em muitas coisas no meu processo de parto, principalmente o que eu deveria fazer. Eu já sabia, mas elas me auxiliaram a tomar banbo, a caminhar. (M13).
\end{abstract}

A Política Nacional de Humanização considera humanização a valorização do sujeito respeitando sua autonomia; as mulheres deste estudo sentiram-se valorizadas em seu processo de parto:

Pediam licença para tocar [as enfermeiras], dizia que não ia doer, tiveram esse cuidado. O jeito de falar e tratar a pessoa, foram educadas. (M2).

Elas me explicam o que é melhor, deram opção para gente escolher. Explicaram tudo direitinho. Em tudo elas [enfermeiras e técnicas de enfermagem] pensaram, até em uma lâmpada, se ficava apagada ou acesa, o que era melhor, elas me perguntaram. (M9).

\section{Categoria 2-Assistência permeada por relações verticais e sentimentos de abandono}

As mulheres identificam a aplicação das tecnologias leves-duras e duras durante o processo de parto. É possível que essas mulheres, com 
base no senso comum, interpretem a realização desses procedimentos como algo estritamente necessário:

A gente deita, alonga, toma banbo de água quente e eu odeio tomar banbo de água quente, principalmente na lombar. E as contrações vinham na lombar. (M10).

Elas [enfermeiras e técnicas de enfermagem] me tiraram da cama que eu estava deitada e disseram para eu fazer agachamentos para eu conseguir parir. (M5).

Eu cheguei e fiquei na bola, depois ela [enfermeira] mandou eu caminhar, tudo o que ela mandava eu fazia. (M1).

Durante o trabalho de parto as mulheres experimentam sentimentos, como desconforto, abandono e negligência:

Eu chamava com educação, mas ninguém aparecia. Enfermeira por favor, vem aqui dar uma olhadinha pra saber como estou, mas ninguém aparecia e eu fiquei sentindo dor sozinha. (M6).

Passei a noite toda sem ninguém chegar perto de mim. Fiquei naquela sala de admissão, frio terrivel, reclamei que estava com frio, ninguém fez nada, passei a noite todinha e não estava tendo assistência, cheguei a querer sair do hospital. (M9).

\section{Discussão}

As mulheres desta pesquisa relatam satisfação com a assistência de Enfermagem, relacionando-a com a atenção no atendimento recebido, no tratamento de acordo com as necessidades individuais e subjetivas, no ficar ao lado e no recebimento de palavras de incentivo durante o processo parturitivo. A satisfação em relação ao parto e nascimento está ligado a fatores culturais, experiências prévias e, sobretudo, à atenção e cuidados prestados durante o trabalho de parto $^{(9)}$.

A assistência de Enfermagem durante o processo de parturição está imbuída por sentimento de apoio, expressado pela ação de segurar na mão, o que propicia na mulher sensação de tranquilidade e estabelece relação de confiança entre as pessoas envolvidas no cenário de parto. Essa relação de confiança mútua desenvolvida entre a Enfermagem e a parturiente favorece o seu bem-estar. Dessa forma, faz-se necessário a criação de vínculo por meio da interação e da capacidade de escuta, para tornar essas relações mais horizontais e terapêuticas, o que inclui também a competência profissional com resolutividade ${ }^{(10)}$.

Foram destacados a aplicabilidade dos métodos não farmacológicos de alívio da dor, banho e deambulação como um dos componentes envolvidos na satisfação das mulheres em relação à assistência de Enfermagem. Esses métodos são práticas que podem ser desenvolvidas pelos profissionais de saúde para proporcionar uma assistência humanizada à parturiente. Tais práticas envolvem respeito e apoio emocional, além de estimular a participação ativa de acompanhantes e das próprias parturientes no processo de parto $^{(11)}$.

O informe consentido da enfermeira para a realização do toque vaginal, a preocupação de aspectos da ambiência que podem gerar desconforto para as mulheres, como a iluminação, foram aspectos abordados nos depoimentos das mulheres. As informações ofertadas pela equipe de Enfermagem, como perguntar em qual posição a parturiente deseja parir, o incentivo à deambulação ou um banho, implica na valorização da sua autonomia em seu trabalho de parto. A oferta de um ambiente aconchegante faz parte de um compromisso que as unidades de saúde devem ter com a ambiência no intuito de proporcionar bem-estar a essas mulheres ${ }^{(12)}$.

É necessário e respeitoso informar à mulher sobre os procedimentos que serão realizados em seu corpo durante o processo parturitivo. Uma relação horizontal entre profissional da saúde e parturientes deve, por conseguinte, proporcionar à mulher a liberdade de escolher ou recusar conscientemente qualquer procedimento relacionado com seu corpo; e esta escolha precisa ser convergente ao seu bem-estar ${ }^{(13)}$.

Valorizar o protagonismo da mulher é uma das maneiras mais eficientes de humanizar a assistência ao parto, aprendendo a compartilhar os saberes e reconhecendo os direitos da mulher em um momento que é inteiramente dela, resgatando os processos fisiológicos no processo de parturição ${ }^{(11)}$. O cuidado à mulher no processo de parto pode revelar cenário de encontros entre profissionais e mulheres, para que ambos 
consigam se colocar um diante do outro em uma relação de existencialidade ${ }^{(14)}$.

Nas relações de cuidado, principalmente na assistência ao parto, historicamente profissionais assumem uma postura de condução do processo de atenção. Esse fato está relacionado também com a liberdade que o trabalhador da saúde tem em seu processo de trabalho. A subjetividade é operadora de realidade, isto é, o modo singular como cada um significa o trabalho e o cuidado influencia o modo como se produz o agir em saúde. Nesse sentido, no cenário em que as mulheres estão fragilizadas, as relações verticais de cuidado podem se expressar de diversas formas, inclusive verticais ${ }^{(15)}$. Dessa forma, identifica-se nesta pesquisa a posição da enfermeira e técnicas de enfermagem como sujeitos que ordenam sentar, tomar banho, caminhar, e a posição da mulher, como um sujeito passivo que realiza o que lhe fora ordenado.

O banho morno é efetivo no alívio da dor durante o trabalho de parto, por proporcionar relaxamento muscular. Entretanto, é um equívoco generalizar esse efeito benéfico para todas as mulheres. Essa prática evidencia a soberania do olhar científico sobre o corpo humano numa relação na qual o corpo é tido como objeto da ciência ${ }^{(16)}$.

A comparação com o atendimento de instituições privadas na fala das mulheres perpassa ao entendimento de que, em hospitais públicos, o parto é indiferenciado, não levando em conta a singularidade de cada mulher. Tem sido amplamente divulgado o descaso com as gestantes que utilizam os serviços públicos ${ }^{(17)}$. A sensação de abandono estava relacionada à expectativa de ter os profissionais mais perto de si, o que não se efetivou. O tratamento negligenciado dos profissionais, somado à falta de informação, evidencia descaso e configura-se como violência institucional. Esta pode se expressar, por exemplo, pela negligência na assistência e omissão de medicação analgésica quando tecnicamente indicada ${ }^{(18)}$.

De modo geral, as mulheres têm sua expectativa construída histórica e socialmente sobre a experiência de parto na maternidade, o que pode imputar em sua percepção valores relativos à (in)satisfação com todo o processo de parto. A ausência de acompanhamento contínuo durante o trabalho de parto, citada pelas mulheres, transcende a satisfação e demonstra fragilidade na assistência. Além de fortalecer uma percepção de abandono nas maternidades, contribui para a insegurança da mulher, visto que, caso esse atendimento não ocorra em tempo oportuno, expõem ao risco desfechos desfavoráveis ao parto e nascimento, impactando nos índices de mortalidade materna e neonatal ${ }^{(18)}$.

Não obstante a pesquisa ter sido desenvolvida em duas maternidades, a primeira delas passou por processo de reforma do centro obstétrico passando a receber somente pacientes reguladas e fora do risco obstétrico habitual, limitando a coleta de dados. Essa situação nos possibilitou dar continuidade à coleta de dados em outra maternidade. Outra limitação deveu-se à pesquisa não ter se debruçado na análise documental dos prontuários, o que auxiliaria diferenciar a assistência da enfermeira obstetra para justificar as condutas implementadas. Entretanto, os achados deste estudo podem subsidiar reflexões acerca dos elementos que envolvem a assistência à mulher no processo parturitivo.

\section{Conclusão}

A percepção das mulheres sobre a assistência de Enfermagem recebida durante o processo de parto normal foi dicotômica, permeada por satisfação, atrelada ao atendimento recebido pela equipe quando este proporcionou bem-estar mediante o desenvolvimento de relação de confiança, existencialidade, oferta de apoio, palavras de incentivo e utilização dos métodos não farmacológicos para alívio da dor no processo de parto.

Entretanto, houve achados de insatisfação relacionada à verticalização das relações, ausência de acompanhamento profissional e banalização da dor durante o trabalho de parto. Essa problemática precisa ser constantemente discutida por demonstrar fragilidade na assistência e expor mulheres e neonatos a desfechos 
inesperados no processo parturitivo, inclusive com recorte de gênero, visto que essas mulheres estão mais vulneráveis à violação de direitos.

\section{Colaborações:}

1 - concepção, projeto, análise e interpretação dos dados: Aiara Nascimento Amaral Bomfim, Telmara Menezes Couto, Keury Thaisana Rodrigues dos Santos Lima, Lais Teixeira da Silva Almeida, Gleice de Oliveira Santos e Ariane Teixeira de Santana;

2 - redação do artigo e revisão crítica relevante do conteúdo intelectual: Aiara Nascimento Amaral Bomfim, Telmara Menezes Couto, Keury Thaisana Rodrigues dos Santos Lima, Lais Teixeira da Silva Almeida, Gleice de Oliveira Santos e Ariane Teixeira de Santana;

3 - aprovação final da versão a ser publicada: Aiara Nascimento Amaral Bomfim, Telmara Menezes Couto, Keury Thaisana Rodrigues dos Santos Lima, Lais Teixeira da Silva Almeida, Gleice de Oliveira Santos e Ariane Teixeira de Santana.

\section{Referências}

1. Bongaarts J. WHO, UNICEF, UNFPA, World Bank Group, and United Nations Population Division Trends in Maternal Mortality: 1990 to 2015 Geneva: World Health Organization, 2015. Popul Dev Rev. 2016;42(4):726-6. DOI: https://doi.org/10.1111/ padr. 12033

2. Barreto ESS, Oliveira JS, Araújo AJS, Queiroz PES, Schulz RS. Redução da mortalidade materna e atuação do enfermeiro. Rev Enferm Contemp. 2018;7(1):20-6. DOI: http://dx.doi.org/ 10.17267/2317-3378rec.v7i1.1370

3. Amaral RCS, Alves VHP, Audrey V, Rodrigues DP, Silva LA, Marchiori GRS. A inserção da enfermeira obstétrica no parto e nascimento: obstáculos em um hospital de ensino no Rio de Janeiro. Esc Anna Nery. 2019;23(1):e20180218. DOI: https://doi. org/10.1590/2177-9465-ean-2018-0218

4. Santana AT, Felzemburgh RDM, Couto TM, Pereira LP. Atuação de enfermeiras residentes em obstetrícia na assistência ao parto. Rev Bras Saude Mater Infant. 2019;19(1):135-44. DOI: https://doi.org/10.1590/1806-93042019000100008
5. Félix HCR, Silva ACL, Matias TGC, Parreira BDM, Silva SR, Ruiz MT. A percepção de gestantes sobre os diferentes tipos de parto. Enferm Brasil. 2018;17(5):497-503. DOI: http://dx.doi. org/10.33233/eb.v17i5.2125

6. Rocha FAA, Fontenele FMC, Carvalho IR, Rodrigues IDCV, Sousa RA, Ferreira Júnior AR. Care during labor and birth: mothers' perception. Rev Rene. 2015;16(6):782-9. DOI: https://doi. org/10.15253/2175-6783.2015000600003

7. Ramos WMA, Aguiar BGC, Conrad D, Pinto CB, Mussumeci PA. Contribution of obstetric nurse in good practices of childbirth and birth assistance. Rev Fund Care Online. 2018;10(1):173-9. DOI: http://dx.doi.org/10.9789/2175-5361.2018. v10i1.173-179

8. Bardin L. Análise de conteúdo. Lisboa: Edições 70; 2016.

9. Silva LS, Leão DCMR, Cruz AFN, Alves VH, Rodrigues DP, Bulcão CP. Women's knowledge about the different positions for labor: a contribution for caring. Rev Enferm UFPE. 2016;10(Suppl 4):3531-6. DOI: 10.5205/ reuol.9681-89824-1-ED.1004sup201604

10. Gomes CBA, Dias RS, Silva WGB, Pacheco MAB, Sousa FGM, Loyola CMD. Prenatal Nursing Consultation: Narratives of Pregnant Women and Nurses. Texto contexto enferm. 2019;28:e20170544. DOI: http://dx.doi. org/10.1590/1980-265X-TCE-2017-0544

11. Melo BM, Gomes LFS, Henriques ACPT, Lima SKM, Damasceno AKC. Implementation of good practice in assistance to labor at a reference maternity. Rev Rene. 2017;18(3):376-82. DOI: $10.15253 / 2175-6783.2017000300013$

12. Silva ALA, Mendes ACG, Miranda GMD, Souza WV. A qualidade do atendimento ao parto na rede pública hospitalar em uma capital brasileira: a satisfação das gestantes. Cad Saúde Pública. 2017;33(12):e00175116. DOI: http:// dx.doi.org/10.1590/0102-311x00175116

13. Bezerra HS, Melo TFV, Oliveira DA. Satisfaction of women with nursing care during the prepartum period. J Nurs UFPE on line. 2017;11(5):1852-7. DOI: 10.5205/reuol.1107798857-1-SM.1105201714

14. Oliveira PS, Couto TM, Lima KTRS, Barral FE, Bomfim ANA. O cuidado por profissionais nas práticas de saúde em obstetrícia à luz de Ayres 
e Buber. Cogitare Enferm. 2019;24:e59889. DOI: http://dx.doi.org/10.5380/ce.v24i0.59889

15. Santos DS, Mishima SM, Merhy EE. Processo de trabalho na Estratégia de Saúde da Família: potencialidades da subjetividade do cuidado para reconfiguração do modelo de atenção. Ciênc saúde coletiva. 2018;23(3):861-70. DOI: https://doi.org/10.1590/1413-81232018233. 03102016

16. Foucault M. Vigiar e punir: nascimento da prisão. Tradução de Raquel Ramalhete. Petrópolis: Vozes; 2014.

17. Andrade PON, Silva JQP, Diniz CMM, Caminha MFC. Fatores associados à violência obstétrica na assistência ao parto vaginal em uma maternidade de alta complexidade em Recife, Pernambuco. Rev Bras Saúde Mater Infant. 2016;16(1):29-37. DOI: https://doi.org/10. 1590/1806-93042016000100004

18. Nascimento SL, Pires VMMM, Santos NA, Machado JC, Meira LS, Palmarella VPR. Conhecimentos e experiências de violência obstétrica em mulheres que vivenciaram a experiência do parto. Enfermería Actual Costa Rica. 2019;(37):66-79. DOI: http://dx.doi.org/10.15517/ revenf.v0ino.37.35264

Recebido: 29 de setembro de 2020

Aprovado: 12 de janeiro de 2021

Publicado: 5 de fevereiro de 2021

A Revista Baiana de Enfermagem utiliza a Licença Creative Commons - Atribuição-NãoComercial 4.0 Internacional.

https://creativecommons.org/licenses/by-nc/4.0/

Este artigo é de acesso aberto distribuído sob os termos da Licença Creative Commons (CC BY-NC).

Esta licença permite que outros remixem, adaptem e criem a partir do seu trabalho para fins não comerciais. Embora os novos trabalhos tenham de lhe atribuir o devido crédito e não possam ser usados para fins comerciais, os usuários não têm de licenciar esses trabalhos derivados sob os mesmos termos. 